\title{
Does Cyg OB2 harbour any open cluster?
}

\author{
E. Bica ${ }^{1}$, Ch. Bonatto ${ }^{1}$, and C. M. Dutra ${ }^{2,3}$ \\ 1 Universidade Federal do Rio Grande do Sul, Instituto de Física, CP 15051, Porto Alegre 91501-970, RS, Brazil \\ 2 Universidade de São Paulo, Instituto de Astronomia, Geofísica e Ciências Atmosféricas, CP 3386, São Paulo 01060-970, SP, \\ Brazil \\ ${ }^{3}$ Universidade Estadual do Rio Grande do Sul, Unidade São Borja, Rua Bompland 512, São Borja 97670-000, RS, Brazil
}

Received 21 November 2002 / Accepted 6 May 2003

\begin{abstract}
We report the detection of three new open cluster candidates located in Cygnus. Two of them are projected near the center of the rich compact association Cyg OB2. These two objects have been found during an optical inspection of that region with Digitized Sky Survey images. Using 2MASS photometry we determined their fundamental parameters. We assumed an age of $1-4 \mathrm{Myr}$ and obtained a distance from the Sun $d_{\odot}=1.8 \pm 0.2 \mathrm{kpc}$ for both objects. Reddening values for Objects 1 and 2 are $E(B-V)=2.12 \pm 0.06$ and $E(B-V)=1.82 \pm 0.06$, respectively. A normal luminosity function is observed down to A0 stars, similar to that of NGC 6910. The two open cluster candidates may form a physical pair in Cyg OB2, similar to $\operatorname{Tr} 14 / \operatorname{Tr} 16$ in Car OB1. Subsequently, we carried out a systematic search with 2 MASS in a region $3^{\circ} \times 3^{\circ}$ encompassing Cyg OB2. A third uncatalogued object resulted from this search which is projected just outside Cyg OB2.
\end{abstract}

Key words. open clusters and associations: general

\section{Introduction}

The Cygnus $\mathrm{X}$ area is a projected, extended structure to which are associated optical, infrared and radio objects (e.g. Dickel et al. 1969; Wendker 1970; Odenwald \& Schwartz 1993). It encompasses one of the richest concentrations of star forming regions in the Galaxy as a result of the Local Arm plunge into Cygnus and/or the existence of a coherent structure with enhanced star formation. Comerón \& Torra (2001) carried out a near-infrared imaging survey of compact H II regions in Cygnus $\mathrm{X}$ using data from Observatorio del Teide and found new embedded clusters. Dutra \& Bica (2001) found new embedded clusters and stellar groups in the direction of known optical and radio nebulae in the Galaxy, including the Cygnus X area, using the Two Micron All Sky Survey (hereafter 2MASS, Skrutskie et al. 1997). Embedded stellar groups are less dense than clusters but appear to be physical systems (Bica et al. 2003). Dutra \& Bica (2001) studied colour-magnitude diagrams (CMDs) using the 2MASS Point Source Catalogue and obtained distances in the range $1.0<d_{\odot}(\mathrm{kpc})<1.8$ for 7 Cygnus X clusters. Recently, Le Duigou \& Knödlseder (2002), also using 2MASS observations, studied the morphology and stellar content of 22 embedded clusters in Cygnus X, some of them new. All these findings correspond to infrared clusters and stellar groups (Bica et al. 2003). An interesting issue is whether any optical or infrared open cluster remains uncatalogued in the area.

Send offprint requests to: Ch. Bonatto, e-mail: charles@if.ufrgs.br
The Cygnus $\mathrm{X}$ area contains the rich compact association Cyg OB2, which has been compared to populous LMC blue clusters in terms of its properties (Knödlseder 2000). The stellar content of the Cyg OB2 association has been much explored, especially in its central parts (e.g. Schulte 1958; Massey \& Thompson 1991; Massey et al. 1995; Knödlseder 2000; Comerón et al. 2002).

Many associations are known to contain star clusters, e.g. NGC 6823 in Vul OB1, NGC 6871 in Cyg OB3, Berkeley 86 in Cyg OB1 (Massey et al. 1995). Carina OB1 contains several clusters, in particular the prominent pair $\operatorname{Tr} 14 / \operatorname{Tr} 16$. An interesting issue is whether $\mathrm{Cyg} \mathrm{OB} 2$, as a prominent association, might harbour any open cluster.

A large number of $\mathrm{O}$ stars has been found in Cyg OB2 spectroscopically or photometrically (Massey \& Thompson 1991; Knödlseder 2000). A recent study (Comerón et al. 2002) estimates this number as 90-100.

Another key issue is the actual distance to the association. Hutchings (1981) assumed an absolute distance modulus ( $m-$ $M)=10.7$, converting to $d_{\odot}=1.38 \mathrm{kpc}$. Humphreys (1978) adopted $d_{\odot}=1.82 \mathrm{kpc}$, while Torres-Dodgen et al. (1991) and Massey \& Thompson (1991) determined $d_{\odot}=1.74 \mathrm{kpc}$ $\left((m-M)_{0}=11.2\right)$. Knödlseder (2000) assumed a distance $d_{\odot}=1.7 \mathrm{kpc}$. The possibility of clusters related to Cyg OB2 in the present study may help constrain the distance. Early reddening determinations have been based on less-reddened stars, e.g. $A_{V}=3.3$ (Hutchings 1981), while more recent studies show a range of values, e.g. Knödlseder (2000) found $A_{V} \approx 5-20$. The large number of main sequence or evolved 
O stars constrains the age to the range 1-4 Myr (Massey et al. 1995).

Any new cluster in the area would bring further constraints to our understanding of the Cygnus X structure and Cyg OB2. In the present study we report two new open cluster candidates in the Cyg OB2 area and perform the first estimate of fundamental parameters for these objects using 2MASS data. In Sect. 2 we present the two new open cluster candidates in Cyg OB2. In Sect. 3 we derive the parameters for these objects from the 2MASS $J, H$ and $K_{\mathrm{S}}$ CMD analysis. In Sect. 4 we present a 2 MASS survey in the area providing a third uncatalogued object, projected just outside Cyg OB2. In Sect. 5 we discuss the results. Finally, in Sect. 6 concluding remarks are given.

\section{New open clusters in Cyg OB2?}

Since Cyg OB2 is a dense and rich association, we were motivated to examine its optical structure on Digitized Sky Survey images. This inspection revealed two objects resembling open clusters which are not listed in any optical open cluster catalogue, e.g. Alter et al. (1970), Lyngå (1987), and more recently Dias et al. (2002). The two objects are projected close to each other $\left(\approx 6^{\prime}\right)$ suggesting a physical pair. The southern cluster candidate, which we refer to as Object 1 , is located at $\mathrm{J} 2000.020^{\mathrm{h}} 33^{\mathrm{m}} 10^{\mathrm{s}}+41^{\circ} 13^{\prime} 07^{\prime \prime}\left(\ell=80.14^{\circ}, b=0.74^{\circ}\right)$ and has angular dimensions of $\approx 3^{\prime} \times 2.5^{\prime}$. The northern companion, Object 2 , is located at $\mathrm{J} 2000.020^{\mathrm{h}} 33^{\mathrm{m}} 15^{\mathrm{s}}+41^{\circ} 18^{\prime} 45^{\prime \prime}$ $\left(\ell=80.22^{\circ}, b=0.79^{\circ}\right)$ and has $\approx 5^{\prime} \times 3^{\prime}$. Object 1 is approximately centered on Cyg OB2. Both objects might be faced as the association core, if located at the same distance.

It is noteworthy that the present objects have been overlooked in previous investigations, despite the fact that both were present in charts and optical images. The northern object detaches as a concentration in Fig. 2 of Schulte (1958) and in Fig. 9 of Massey \& Thompson (1991). Both objects are clear in zone 3 of Fig. 1 of Massey \& Thompson (1991) - a blue POSS plate. Le Duigou \& Knödlseder (2002) have not investigated the central zone of Cygnus OB2 on the basis of having already covered it in sufficient detail in Knödlseder (2000). The work by Dutra \& Bica (2001) studied directions of prominent nebula, but none occurs in the center of Cyg OB2.

We show in Figs. 1 and 2 red XDSS images of Objects 1 and 2, respectively, in which they clearly detach from the background. They are also visible in blue XDSS images, which suggests that reddening is not extreme. Finally, in Figs. 3 and 4 we show these objects in 2MASS $K_{\mathrm{s}}$ extractions. They are also prominent, suggesting that they are neither field fluctuations nor a variable absorption effect. The crosses in Figs. 3 and 4 indicate artifacts on the 2MASS images.

\subsection{The 2MASS photometry}

The photometric errors as a function of magnitude are shown in e.g. Soares \& Bica (2002) for the three bands $J, H$ and $K_{\mathrm{s}}$ which are available in 2 MASS (second incremental data release at http://www.ipac.caltech.edu/2mass/releases/ second/). From their Fig. 5, one can see that errors become

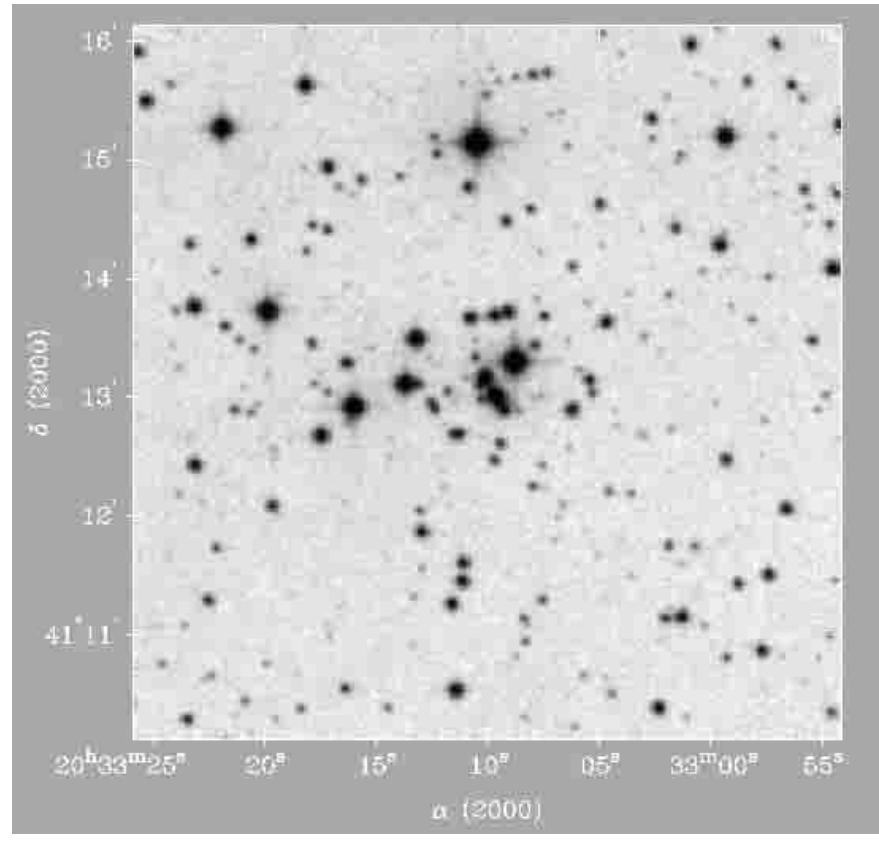

Fig. 1. $6^{\prime} \times 6^{\prime}$ XDSS R image of Object 1 .

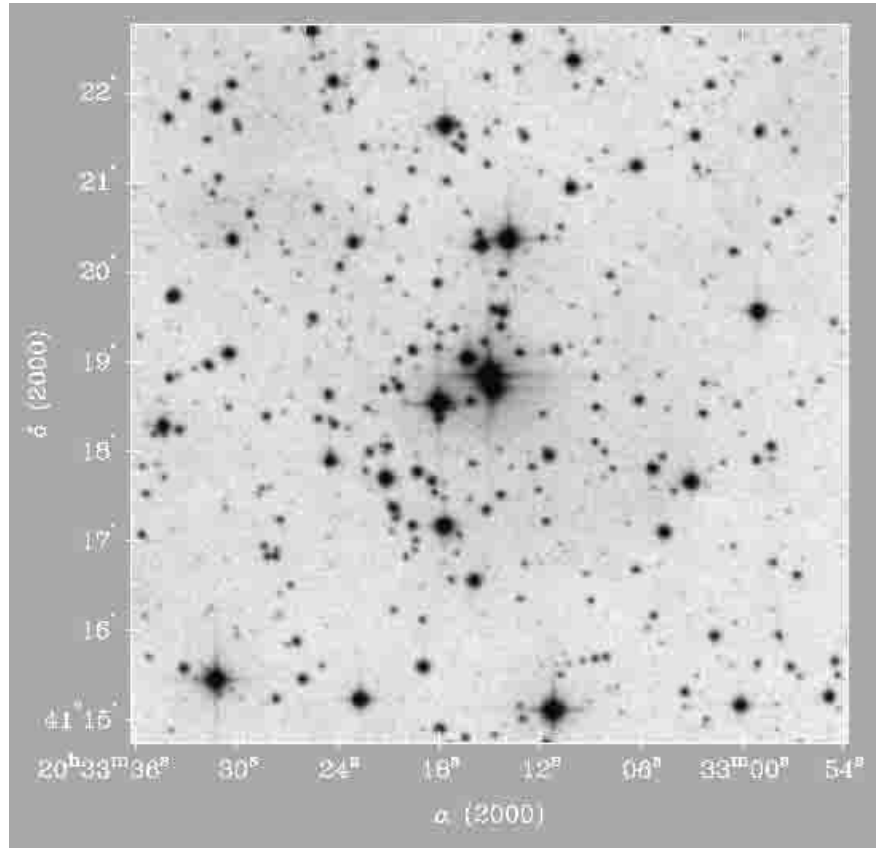

Fig. 2. $8^{\prime} \times 8^{\prime}$ XDSS R image of Object 2 .

important for stars fainter than $\approx 13.5\left(K_{\mathrm{s}}\right)$ and $\approx 15(J)$, while $H$ has an intermediate behaviour.

For Object 1 we made circular extractions centered on the Sect. 1 coordinates with $2.0^{\prime}$ radius, while for Object 2 we used a $2.5^{\prime}$ radius. Comparison fields have been extracted with the same radii as those used for the objects at East and West of both objects, with center to center distances of $5.0^{\prime}$ and $6.0^{\prime}$, respectively for Object 1 and Object 2. Extractions have been performed using the VizieR tool at http://vizier.u-strasbg.fr/viz-bin/VizieR? - source $=2$ MASS. The results are shown in Figs. 5 and 6, respectively for Objects 1 and 2. The top panel of each figure 


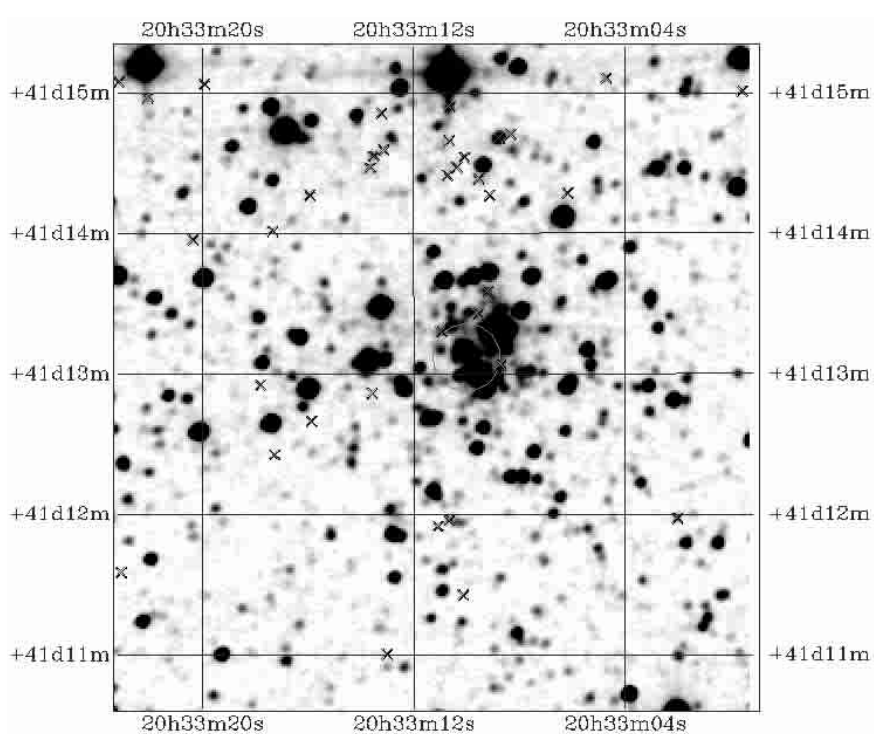

Fig. 3. $5^{\prime} \times 5^{\prime} 2$ MASS $K_{\mathrm{s}}$ image of Object 1 .

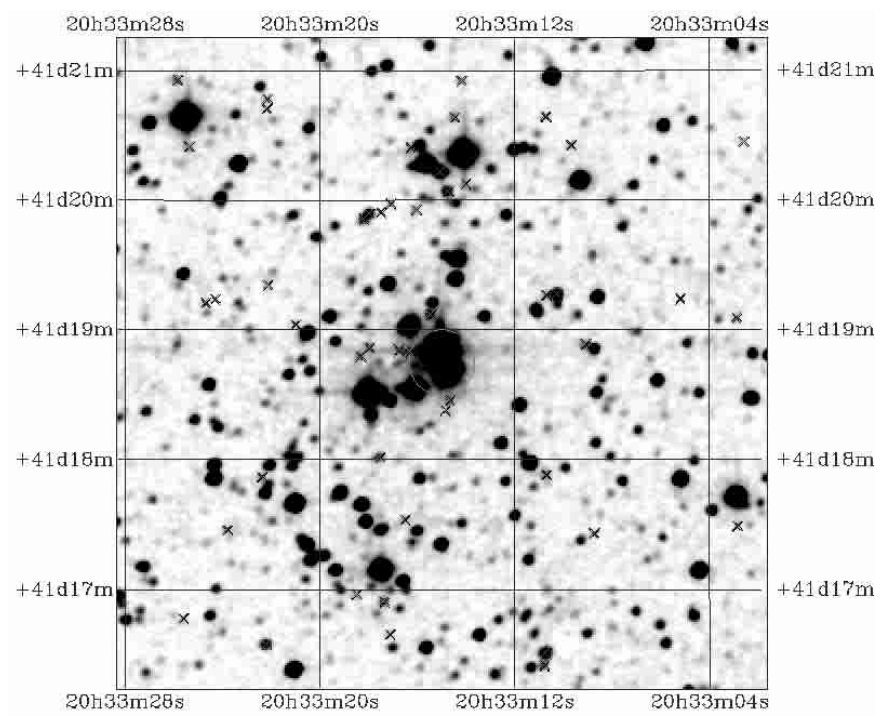

Fig. 4. $5^{\prime} \times 5^{\prime} 2$ MASS $K_{\mathrm{s}}$ image of Object 2 .

deals with the object extraction, while the East and West offset fields are in the middle and bottom panels, respectively. Left panels show $J \times(J-H)$ and right panels $K_{\mathrm{S}} \times\left(J-K_{\mathrm{S}}\right)$ CMDs.

In both cases a prominent main sequence (MS) in the CMDs of the objects stands out as compared to those of the adjacent fields.

\subsection{Available spectroscopy and optical photometry}

Spectroscopic and optical photometric data are available for several stars in the area of each cluster candidate. Considering Schulte's (1958) and Massey \& Thompson's (1995) stars, designated respectively by $\mathrm{S}$ and MT, the stars $\mathrm{S} 8 \mathrm{~A}=\mathrm{MT} 465$, $\mathrm{S} 8 \mathrm{~B}=\mathrm{MT} 462, \mathrm{~S} 8 \mathrm{C}=\mathrm{MT} 483, \mathrm{~S} 8 \mathrm{D}=\mathrm{MT} 473, \mathrm{MT} 441$, MT 507 and MT 457 are in the area of Object 2. In the area of Object 1, there occur the stars S $9=$ MT 431, MT 417, MT 455, MT 477 and MT 488.

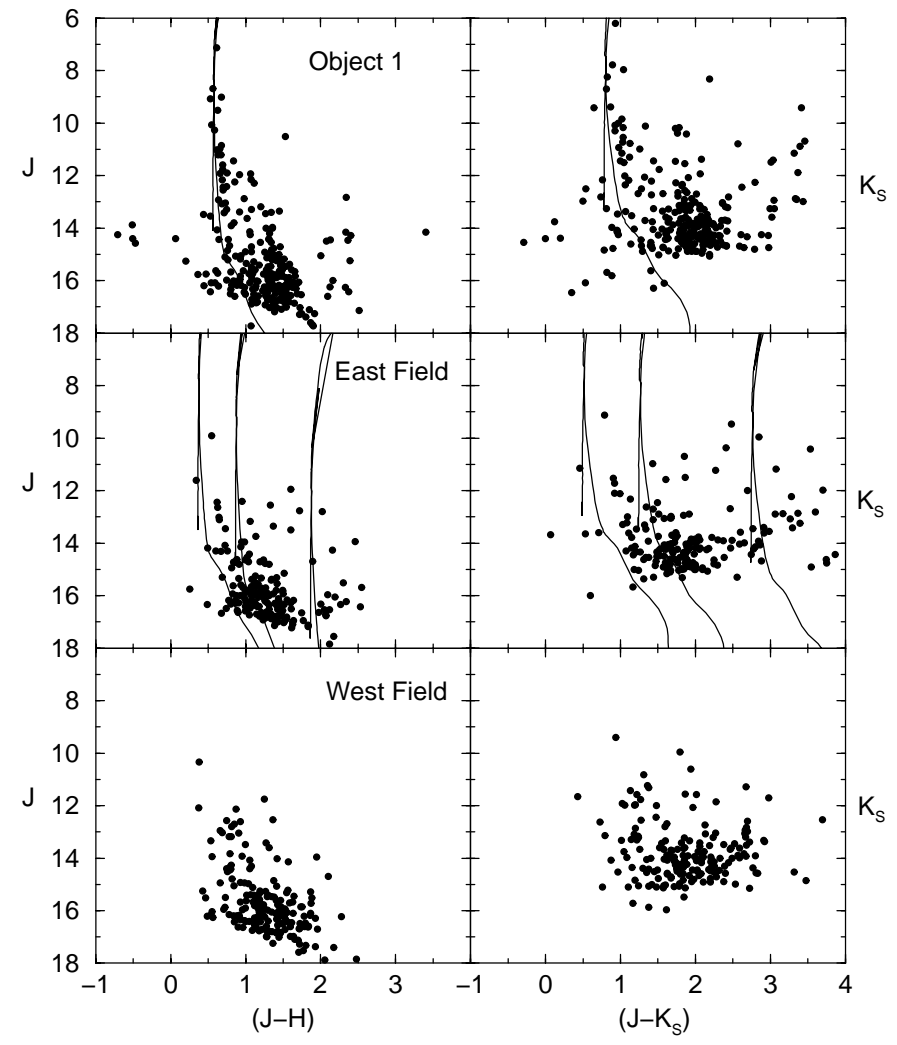

Fig. 5. $J \times(J-H)\left(\right.$ left panel) and $K_{\mathrm{S}} \times\left(J-K_{\mathrm{S}}\right)$ (right panel) CMDs for Object 1; corresponding East and West offset fields are also shown. We show a 4 Myr Padova isochrone at $(m-M)_{0}=11.2$. In the upper panels, the values $E(J-H)=0.70 \pm 0.02, E\left(J-K_{\mathrm{S}}\right)=1.04 \pm 0.02$, $A_{J}=1.99 \pm 0.05$ and $A_{K_{\mathrm{S}}}=0.81 \pm 0.02$ have been derived. In the middle panels, we assumed $A_{V}=5,10$ and 20 .

Massey \& Thompson (1991) condensed previous spectral type determinations (Schulte 1958; Hutchings 1981; Walborn 1973) and their own. In Object 2, S 8A, S 8B, S 8C and MT 457 are evolved O stars. S 8D is an O8V, and MT 507 is an $08.5 \mathrm{~V}$. In Object 1, S 9 and MT 417 appear to be evolved O stars while MT 455 is an O8V. We remark the high concentrations of $\mathrm{O}$ stars in these small areas, which might be signatures of open clusters. Concerning the recent infrared spectral classifications by Comerón et al. (2002), only one star (B19) is in the area of the present objects. This star is the same as S 9 in the optical papers above. Its classification as emission-line star in the infrared is consistent with its evolved $\mathrm{O}$ star nature.

\subsection{Structural analysis of the cluster candidates}

Figure 7 shows the radial distribution of star density in and around the cluster candidates. Before counting stars, we applied a cutoff $(J<15.5)$ to the objects and offset fields to avoid undersampling, i.e. to avoid spatial variations in the number of faint stars which are numerous, affected by large errors, and may include spurious detections, in the area of the objects. Colour filters have not been applied, since tests revealed them to be unnecessary. For the objects we used concentric annuli with a step of $0.5^{\prime}$ in radius up to $5^{\prime}$ in radius. In the region $3.5^{\prime}-4.0^{\prime}$, both clusters show up in the respective profiles, thus 


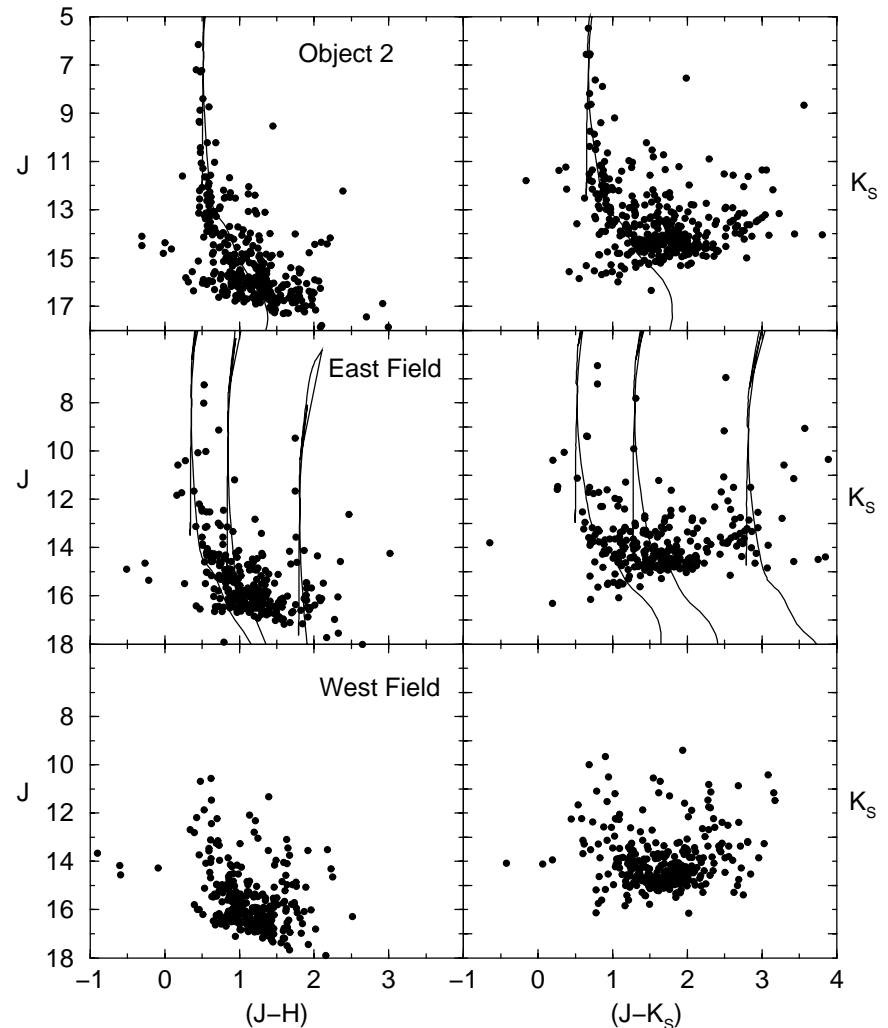

Fig. 6. Same as Fig. 5 for Object 2. Values derived from the upper panels are $E(J-H)=0.64 \pm 0.02, E\left(J-K_{\mathrm{S}}\right)=0.91 \pm 0.02, A_{J}=$ $1.83 \pm 0.05$ and $A_{K_{\mathrm{S}}}=0.75 \pm 0.02$.

it is not useful to measure annuli for $R>3.0^{\prime}$ since the objects form a projected pair nearly at that distance, and contamination would result from the companion.

We also show the background levels which are the average of the East and West offset fields of each object. Object 1 presents a prominent core of radius $\approx 1^{\prime}$ surrounded by a halo extending at least up to radius $2^{\prime}$.

Object 2 has a deficiency of stars near the center, as can also be seen in Fig. 2, but beyond this radius it presents significant star excesses over the background. Besides, its CMD (Fig. 6) presents a MS which suggests its nature as a cluster. The central deficiency might in part be accounted for by faint star images blended to the several bright ones near the object center. The limiting radius appears to be $\approx 2.5^{\prime}$.

\section{Fundamental parameters}

\subsection{Distance}

Since spectroscopic data for member stars are available, we use them to derive accurate distance values. Following Sect. 2.1, we restrict to the MS stars, since evolved early-type stars vary fast their luminosity. In Object 1 we have the O8V star MT 455, while in Object 2 there occur the O8V star S 8D and the $08.5 \mathrm{~V}$ star MT 507. We adopted as intrinsic magnitudes and colours those of Binney \& Merrifield (1998). We remind that the latter set employs the $K$ filter, while our observations correspond to the 2MASS $K_{\mathrm{S}}$ filter. We will assume their equivalence, since

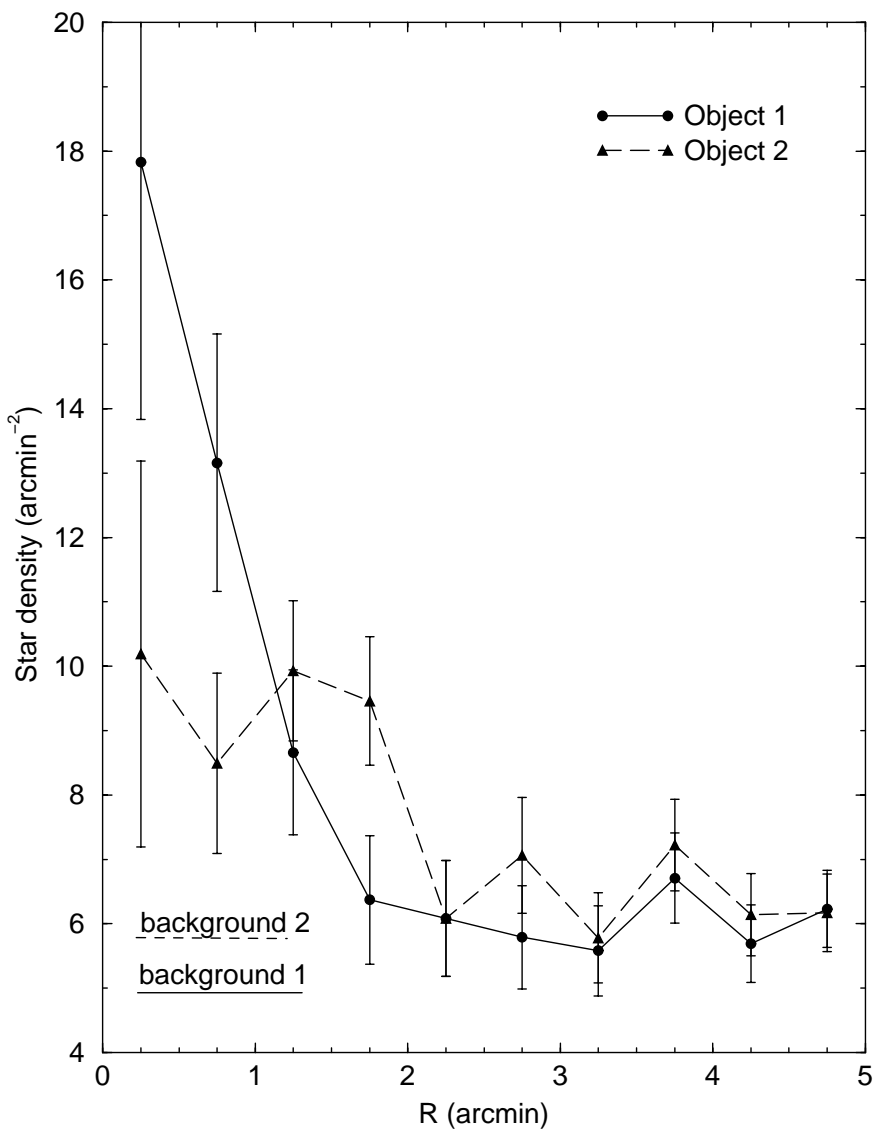

Fig. 7. Radial distribution of surface star density for the two objects. Background levels are averages of the East and West offset positions and are shown as a continuous line (Object 1) and dashed line (Object 2). Notice that a magnitude cutoff $(J<15.5)$ has been applied to the objects and offset fields.

magnitudes in both filters may differ by $\approx 0.02$ (Girardi 2003 private communication).

Since the total-to-selective absorption $\left(R_{V}\right)$ increases for star-forming regions (e.g. Vrba et al. 1991), we test $R_{V}=3.2$ and 3.6 to compute distances. Adopting $\frac{A_{\lambda}}{A_{V}}$ for $J$ and $H$ from Schlegel et al. (1998) and the ratio $\frac{A_{K_{S}}}{A_{V}}=0.118$ from Dutra et al. (2002), the following relations can be derived: $A_{J}=$ $0.893 R_{V} E(J-H)$ and $A_{K_{\mathrm{S}}}=0.242 R_{V} E\left(J-K_{\mathrm{S}}\right)$.

Table 1 summarizes the computations for the above object stars. The data for the stars in the optical are from Massey \& Thompson (1991) and in the infrared from 2MASS.

Distances derived from optical photometry are very dependent on the assumed $R_{V}$. In the infrared the resulting distance values are more constrained, indicating that the actual $R_{V}$ is $\approx 3.2$ (Table 1 ). In fact, $R_{V}=3.2$ gives the most consistent results between the different distance estimates. Considering the absolute distance moduli and distances calculated with $R_{V}=3.2, \mathrm{~S} 8 \mathrm{D}$ gives $(m-M)_{0}=11.32 \pm 0.12$ and $d_{\odot}=$ $1.83 \pm 0.10 \mathrm{kpc}$, MT 507 gives $(m-M)_{0}=11.57 \pm 0.11$ and $d_{\odot}=2.06 \pm 0.11 \mathrm{kpc}$, and finally, MT 455 gives $(m-M)_{0}=$ $11.22 \pm 0.20$ and $d_{\odot}=1.76 \pm 0.16 \mathrm{kpc}$. The two stars in Object 2 give $(m-M)_{0}=11.45 \pm 0.18$ and $d_{\odot}=1.95 \pm$ $0.17 \mathrm{kpc}$. Uncertainties correspond to different estimates based 
Table 1. Optical and infrared parameters for the MS stars in Objects 1 and 2.

\begin{tabular}{ccccccccccccc}
\hline \hline Object & Star & Type & $R_{V}$ & $V$ & $(B-V)$ & $(B-V)_{0}$ & $E(B-V)$ & $A_{V}$ & $V_{0}$ & $M_{V}$ & $(m-M)_{0}$ & $d(\mathrm{kpc})$ \\
\hline 2 & S 8D & O8V & 3.2 & 12.02 & 1.45 & -0.32 & 1.77 & 5.66 & 6.36 & -4.9 & 11.26 & 1.8 \\
2 & S 8D & O8V & 3.6 & 12.02 & 1.45 & -0.32 & 1.77 & 6.37 & 5.65 & -4.9 & 10.55 & 1.3 \\
2 & MT 507 & O8.5V & 3.2 & 12.70 & 1.54 & -0.32 & 1.86 & 5.95 & 6.75 & -4.7 & 11.45 & 1.9 \\
2 & MT 507 & O8.5V & 3.6 & 12.70 & 1.54 & -0.32 & 1.86 & 6.70 & 6.00 & -4.7 & 10.70 & 1.4 \\
1 & MT 455 & O8V & 3.2 & 12.92 & 1.81 & -0.32 & 2.13 & 6.82 & 6.10 & -4.9 & 11.00 & 1.6 \\
1 & MT 455 & O8V & 3.6 & 12.92 & 1.81 & -0.32 & 2.13 & 7.67 & 5.25 & -4.9 & 10.15 & 1.1 \\
\hline & & & & $J$ & $(J-H)$ & $(J-H)_{0}$ & $E(J-H)$ & $A_{J}$ & $J_{0}$ & $M_{J}$ & & 1.23 \\
\hline 2 & S 8D & O8V & 3.2 & 8.88 & 0.47 & -0.17 & 0.63 & 1.83 & 7.05 & -4.2 & 11.23 \\
2 & S 8D & O8V & 3.6 & 8.88 & 0.47 & -0.17 & 0.63 & 2.06 & 6.83 & -4.2 & 11.00 & 1.6 \\
2 & MT 507 & O8.5V & 3.2 & 9.35 & 0.46 & -0.16 & 0.62 & 1.78 & 7.57 & -4.0 & 11.57 & 2.1 \\
2 & MT 507 & O8.5V & 3.6 & 9.35 & 0.46 & -0.16 & 0.62 & 2.00 & 7.35 & -4.0 & 11.35 & 1.9 \\
1 & MT 455 & O8V & 3.2 & 9.07 & 0.53 & -0.17 & 0.70 & 1.99 & 7.08 & -4.2 & 11.26 & 1.8 \\
1 & MT 455 & O8V & 3.6 & 9.07 & 0.53 & -0.17 & 0.70 & 2.24 & 6.83 & -4.2 & 11.01 & 1.6 \\
\hline & & & & $K_{\mathrm{S}}$ & $\left(J-K_{\mathrm{S}}\right)$ & $\left(J-K_{\mathrm{S})_{0}}\right.$ & $E\left(J-K_{\mathrm{S}}\right)$ & $A_{K_{\mathrm{S}}}$ & $K_{\mathrm{S}_{0}}$ & $M_{K_{\mathrm{S}}}$ & \\
\hline 2 & S 8D & O8V & 3.2 & 8.19 & 0.69 & -0.22 & 0.91 & 0.70 & 7.49 & -4.0 & 11.45 & 1.9 \\
2 & S 8D & O8V & 3.6 & 8.19 & 0.69 & -0.22 & 0.91 & 0.79 & 7.40 & -4.0 & 11.36 & 1.9 \\
2 & MT 507 & O8.5V & 3.2 & 8.64 & 0.71 & -0.21 & 0.92 & 0.71 & 7.93 & -3.7 & 11.68 & 2.2 \\
2 & MT 507 & O8.5V & 3.6 & 8.64 & 0.71 & -0.21 & 0.92 & 0.80 & 7.84 & -3.7 & 11.59 & 2.1 \\
1 & MT 455 & O8V & 3.2 & 8.25 & 0.82 & -0.22 & 1.04 & 0.81 & 7.42 & -4.0 & 11.38 & 1.9 \\
1 & MT 455 & O8V & 3.6 & 8.25 & 0.82 & -0.22 & 1.04 & 0.91 & 7.34 & -4.0 & 11.30 & 1.8 \\
\hline
\end{tabular}

on $R_{V}=3.2$. The above values and uncertainties are consistent with $(m-M)_{0}=11.2$ and $d_{\odot}=1.74 \mathrm{kpc}$ by Massey $\&$ Thompson (1991) for Cyg OB2.

We conclude that both objects are part of Cyg OB2, and we will assume the Cyg OB2 distance value for both objects, since it is based on a large number of stars.

\subsection{Age}

The large number of evolved and MS O stars identified spectroscopically (Sect. 2.2) in the present two objects constrains their age to $1-4 \mathrm{Myr}$, likewise the age range adopted for the association itself (Massey et al. 1995).

\subsection{Reddening}

In order to derive parameters for Objects 1 and 2, we use solar metallicity Padova isochrones from Girardi et al. (2002). Taking into account the age range obtained in Sect. 3.2, we adopted the youngest available solar metallicity isochrone in the Padova set, that of $4 \mathrm{Myr}$. For reddening and absorption transformations we use $R_{V}=3.2$ according to Sect. 3.1, and the relations $A_{J}=0.276 A_{V}$ and $A_{K_{\mathrm{S}}}=0.118 A_{V}$ (Sect. 3.1). Finally, the relations $E(J-H)=0.33 E(B-V)$ and $E\left(J-K_{\mathrm{S}}\right)=$ $0.49 E(B-V)$ can be therefrom derived. Accordingly, we adopt an absolute distance modulus $(m-M)_{0}=11.2$ (Sect. 3.1) for both objects and derive for Object $1 E(J-H)=0.70 \pm 0.02$, $E\left(J-K_{\mathrm{S}}\right)=1.04 \pm 0.02, A_{J}=1.99 \pm 0.05$ and $A_{K_{\mathrm{S}}}=0.81 \pm 0.02$, while for Object 2 we find $E(J-H)=0.64 \pm 0.02, E\left(J-K_{\mathrm{S}}\right)=$ $0.91 \pm 0.02, A_{J}=1.83 \pm 0.05$ and $A_{K_{\mathrm{S}}}=0.75 \pm 0.02$.

We show in Figs. 5 and 6 (top panels) Padova isochrone fittings with the above parameters to the two cluster candidates, respectively. The agreement between isochrone and the stellar distributions of the objects favours them as open clusters.
Notice in Figs. 5 and 6 the somewhat poorer fits for $\left(J-K_{\mathrm{S}}\right)$ as compared to that for $(J-H)$. The nominal errors for $A_{K_{\mathrm{S}}}$ above are based on the reddening transformations from the value derived for $E(J-H)$, and appear to be larger when obtained directly from the respective diagram.

A fundamental issue is whether the present structures might be absorption windows in a more reddened region. In addition to the fit of a $4 \mathrm{Myr}$ Padova isochrone with the object parameters above to both CMDs (top panels of Figs. 5 and 6), we test this hypothesis by reddening the $4 \mathrm{Myr}$ isochrone by three different values: $A_{V}=5,10$ and 20 (middle panels). For Objects 1 and 2 , there does not appear to be star sequences justifying this hypothesis.

\subsection{Luminosity functions and masses}

A simple cluster mass comparison can be made by taking into account the number of massive stars and assuming similar mass functions with respect to a reference cluster. According to Sect. 2.1, Object 1 has 3 massive stars (OV or evolved O stars), while Object 2 has 6 . We make a comparison with the Trapezium cluster (Prosser et al. 1994) and NGC 3603 (Moffat 1983; Melnick et al. 1989). The Trapezium has 2 massive stars, while NGC 3603 has 14, including two WR stars. Accordingly, Object 1 would have a mass intermediate between the two reference clusters, while Object 2 would be somewhat more massive than the Trapezium cluster.

Figure 8 depicts the luminosity functions in both $J$ and $K_{\mathrm{S}}$ filters (shaded area) built as the difference of the number of stars in a given magnitude bin between object (continuous line) and offset field (dashed line). These luminosity functions are built after applying a cutoff $(J<15.5)$ to the objects and offset fields. The background-subtracted luminosity function of Object 1 increases up to the limit in $J$ but turns over for the two 


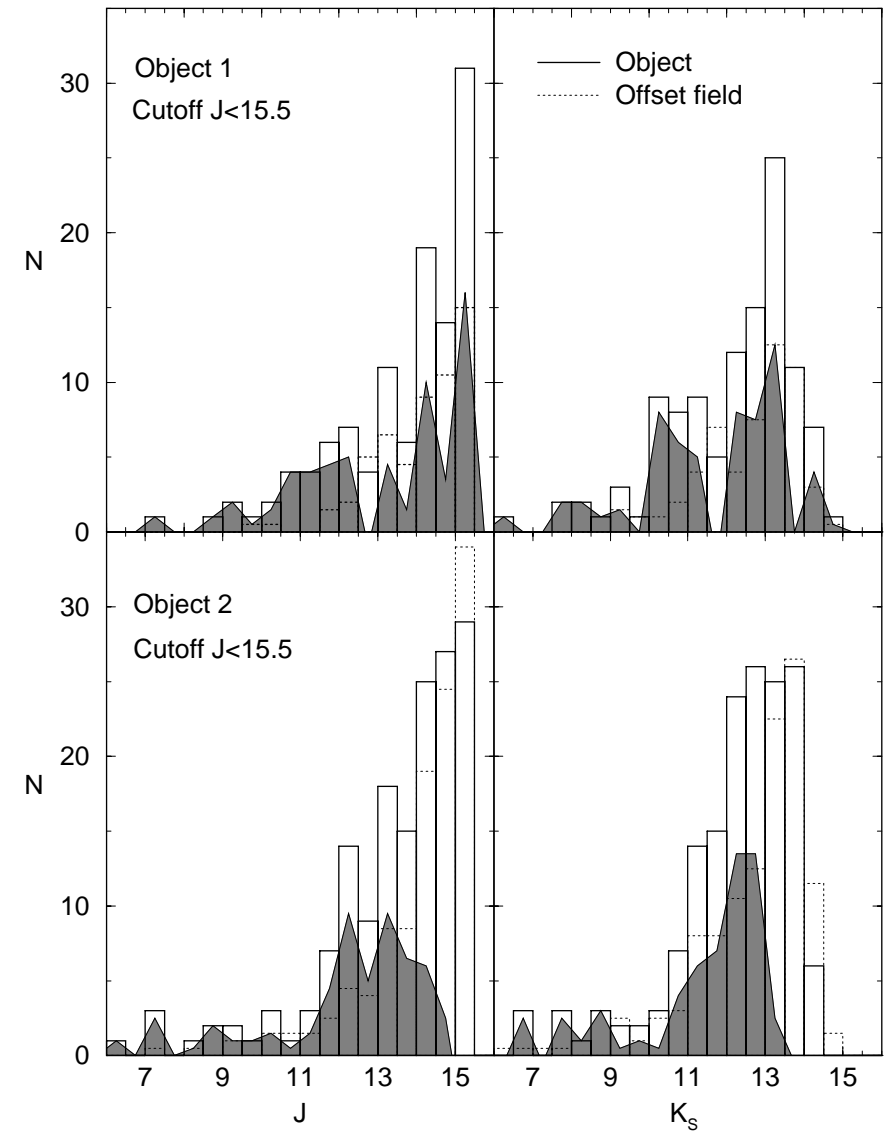

Fig. 8. Luminosity functions for Objects 1 and 2 and offset fields, both for $J$ and $K_{\mathrm{S}}$. Continuous line is object area, dotted line is the offset field; their difference is shown as the shaded area.

last magnitude bins in $K_{\mathrm{S}}$. Object 2 has an increasing function up to $J=13.5$ and $K_{\mathrm{S}}=12.5$.

In order to infer on the low-mass behaviour, we compare the luminosity functions for both $J$ and $K_{\mathrm{S}}$ filters with that of a reference cluster such as NGC 6910. Delgado \& Alfaro (2000) place NGC 6910 at the distance to Cyg OB2, but other studies place it closer, e.g. WEBDA (Mermilliod $1996-$ http: //obswww. unige.ch/webda). However, this distance uncertainty affects little the conclusions below. These studies give $E(B-V) \approx 1$, which converts to $A_{V}=3.2, A_{J}=0.9$ and $A_{K_{\mathrm{S}}}=0.4$. We adopted the distance of Delgado \& Alfaro (2000) and applied the above absorptions to the NGC 6910 data.

Stars in the direction of NGC 6910 have been extracted within a radius of $6.0^{\prime}$ centered on $\mathrm{J} 2000.020^{\mathrm{h}} 23^{\mathrm{m}} 08^{\mathrm{s}}$ $+40^{\circ} 46^{\prime} 30^{\prime \prime}$, including two offset fields of equal dimensions with centers at $17^{\prime}$ at South and North away from the cluster. The same magnitude cutoff $(J<15.5)$ has been applied to NGC 6910 and its offset fields in order to build the 2MASS luminosity function.

The $J$ and $K_{\mathrm{S}}$ luminosity functions of Objects 1 and 2 have been dereddened and brought to absolute magnitudes, and then compared to that of NGC 6910 in Fig. 9, in which we also indicate the loci of MS stars, according to Binney \& Merrifield (1998).

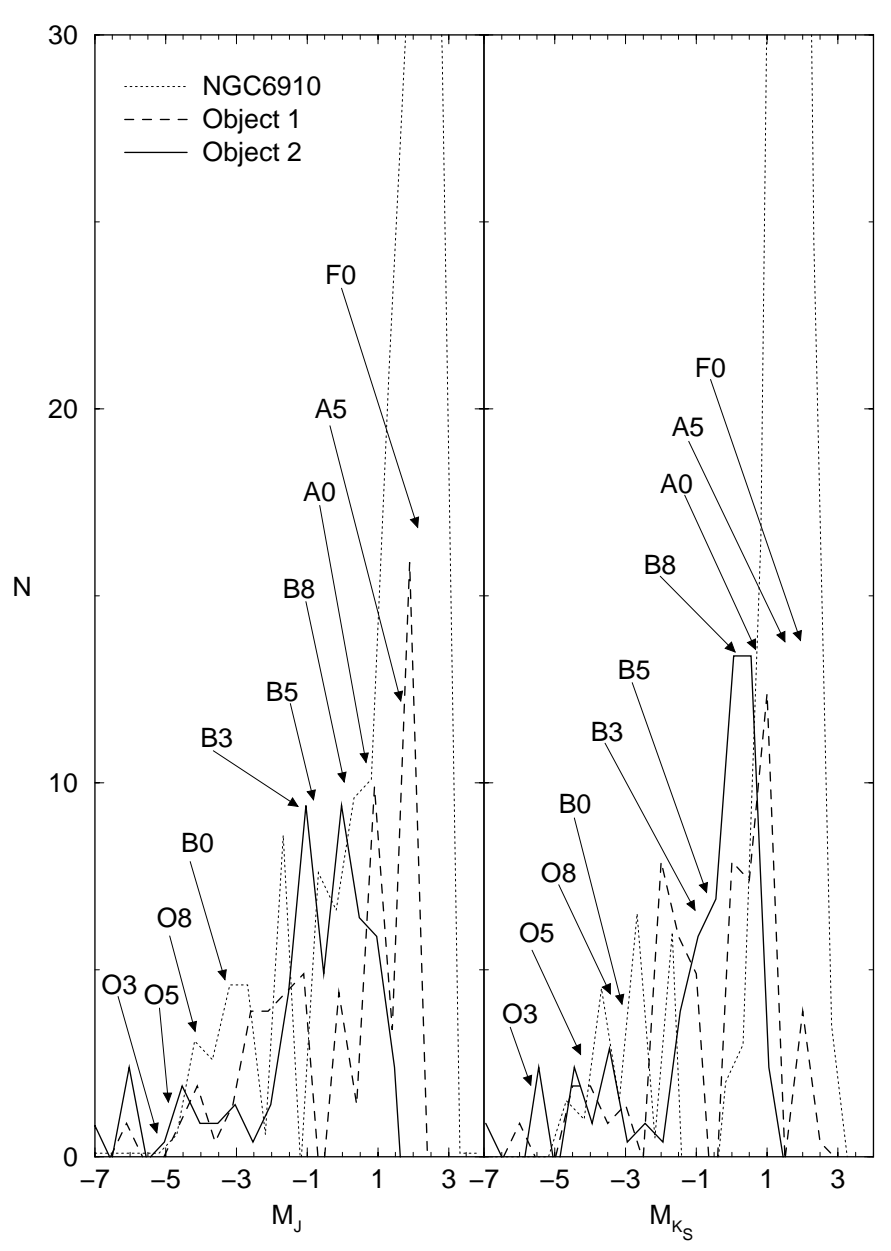

Fig. 9. Absolute luminosity functions: comparison of Object 1 (dashed line) and Object 2 (continuous line) with NGC 6910 (dotted line). Spectral types of MS stars are indicated, according to Binney \& Merrifield (1998).

The three luminosity functions are similar from high-mass down to $\approx \mathrm{A} 0$ stars. The turnovers in the luminosity functions of Objects 1 and 2, with respect to that of NGC 6910, may be due to crowding and completeness effects, since both objects span areas 6-9 times smaller than that of NGC 6910 (angular diameter $12^{\prime}$ ). Alternatively, the turnovers might be caused by an unusual luminosity function characterizing an aggregate of massive and intermediate-mass stars.

\section{The 2MASS $K_{S}$ survey on the Cygnus $X$ area}

In order to verify the existence of other uncatalogued similar objects in the area of $\mathrm{Cyg} \mathrm{OB} 2$, we carried out a systematic visual inspection of a region $3^{\circ} \times 3^{\circ}$ encompassing Cyg OB2 (square area in Fig. 10). We used the All-Sky Release of the 2MASS Atlas and examined $312 K_{\mathrm{S}}$ images of $15^{\prime} \times 7^{\prime}$ which are those least affected by reddening. Dutra \& Bica (2000) carried out a similarly systematic survey encompassing the Galactic center. In case of possible targets, we also examined the $J$ and $H$ images.

We retrieved most of the catalogued objects in the area (Fig. 10) as well as a new object which is located outside 


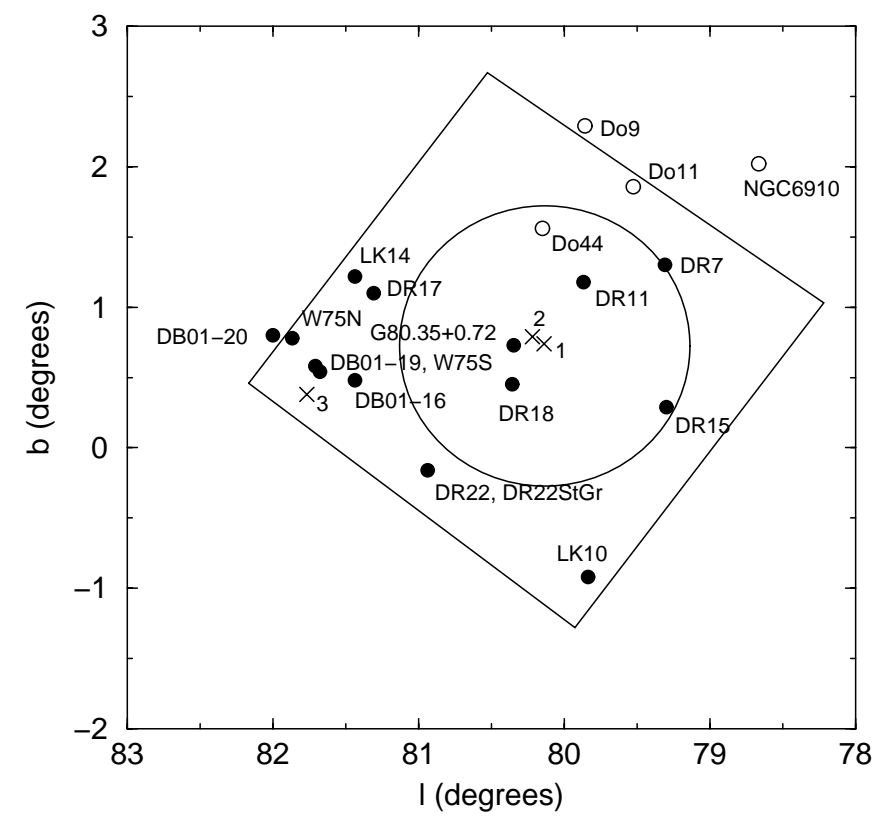

Fig. 10. Angular distribution of the new and previously catalogued objects in the Cyg OB2 area. Large circle is Cyg OB2; filled circles are infrared clusters or stellar groups; open circles are optical open clusters or stellar groups; Objects 1, 2 and 3 are indicated by crosses. Square represents the region surveyed with 2MASS. We only show objects within the square or close to its borders.

Cyg OB2, which we refer to as Object 3. This object does not appear in optical images, indicating high reddening.

We show in Fig. 11 a $K_{\mathrm{S}}$ image of Object 3, where one can see a concentration of stars with a diameter of $\approx 2.5^{\prime}$. Stars have been extracted inside a radius of $1.2^{\prime}$ centered at J2000.0 $20^{\mathrm{h}} 40^{\mathrm{m}} 00^{\mathrm{s}}+42^{\circ} 18^{\prime} 30^{\prime \prime}$, with two offset fields of equal radius centered at 4' away to the North and South. The CMDs of Object 3 and offset fields are given in Fig. 12. The object appears to be a physical system, with a tight sequence of stars in $K_{\mathrm{S}} \times\left(H-K_{\mathrm{S}}\right)$ resembling a giant branch. However, it cannot be excluded a very reddened younger cluster. In order to confirm the physical nature of Object 3 , deeper photometry is necessary to test the possibility of an intermediate age turnoff.

Object 3 is indicated in Fig. 10 together with Objects 1 and 2 and all previously reported objects in the area. Several embedded clusters or stellar groups have been reported in the surroundings of Cyg OB2, as can be seen in the recent catalogue by Bica et al. (2003) which is basically complete to mid 2002. We also included the new objects by Le Duigou \& Knödlseder (2002), which are indicated by LK in Fig. 10. The notation DB01- indicates objects from Dutra \& Bica (2001) while the remaining notations refer to clusters or stellar groups embedded in their radio nebulae. Also included are optical open clusters as catalogued e.g. in Alter et al. (1970). The Cyg OB2 area ( $2^{\circ}$ diameter) is delimited by the circle and the objects projected inside it might be related to itself, to the general field of Cygnus X, or to the Perseus or Outer Arms in the background. The embedded cluster in G 80.35+0.72 (ECX 627 or 18P61), projected close to the nucleus of Cyg OB2, appears to be a background cluster in the Outer Arm (Comerón \& Torra 2001). DR 7 (ECX6-18) might be in the Perseus Arm

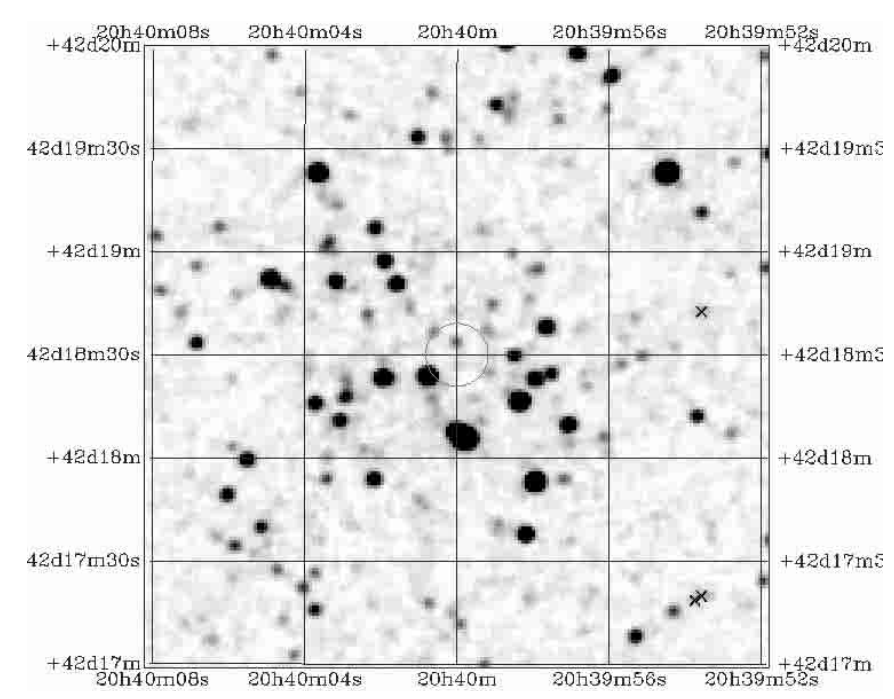

Fig. 11. $3^{\prime} \times 3^{\prime} 2$ MASS $K_{\mathrm{S}}$ image of Object 3 .

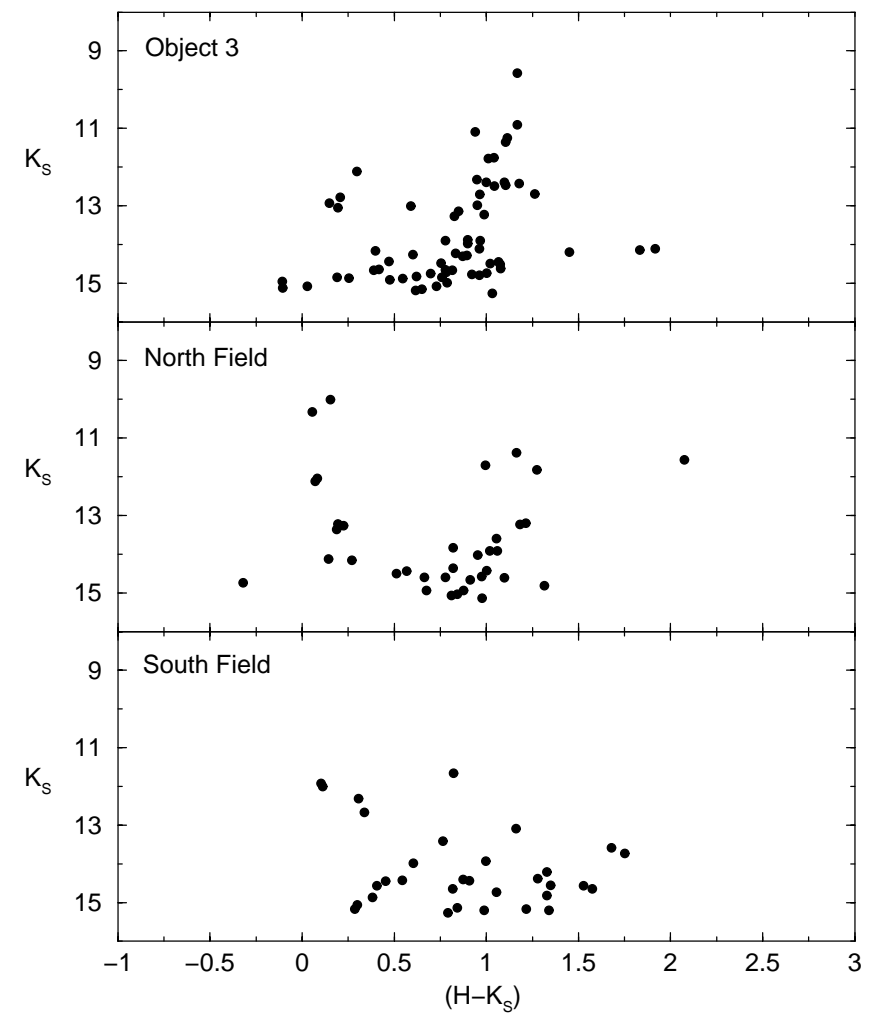

Fig. 12. $K_{\mathrm{S}} \times\left(H-K_{\mathrm{S}}\right)$ CMDs of Object 3 and North and South offset fields. Extraction radius is $1.2^{\prime}$ for object and fields.

(Comerón \& Torra 2001) or in Cygnus X (Dutra \& Bica 2001). DR 15 (IRAS 20306+4005, ECX6-25) is an embedded cluster probably in Cygnus X (Comerón \& Torra 2001), while DR 18 is a loose star-forming region in Cygnus X (Comerón \& Torra 1999). DR 11 (ECX6-21) encloses a stellar group in Cygnus $X$ (Comerón \& Torra 2001). The optical object Do 44 is a loose concentration of stars near the edge of Cyg OB2; it is probably part of Cyg OB2 but does not look like an open cluster. Its Trumpler classification is IV2p, hardly discernible from the background. All these objects assigned to Cygnus X could be related to $\mathrm{Cyg} \mathrm{OB} 2$, however, available distances are not so 
precisely determined. With respect to the present two objects (Sect. 3), they are projected right at the center of Cyg OB2 and consequently more probably related to it, in addition to the fact that a series of massive stars attributed to Cyg OB2 (Sect. 2.2) are part of them.

We conclude from this survey that no other open cluster candidate comparable to Objects 1 and 2 are projected on or located in Cyg OB2.

\section{Discussion}

The analysis by Knödlseder (2000) revealed a spherically symmetric association of $\approx 2^{\circ}$ in diameter with a half-light radius of $13^{\prime}$, corresponding to $R_{\mathrm{h}}=6.4 \mathrm{pc}$ at an assumed distance of $1.7 \mathrm{kpc}$. In the present study we find for both objects a compatible distance of $\approx 1.8 \pm 0.2 \mathrm{kpc}$. We derive for Object 1 $A_{V}=6.6 \pm 0.2$ and for Object $2 A_{V}=5.6 \pm 0.2$, thus they belong to the less reddened parts of the association. The Object 1 position basically coincides with the Cyg OB2 center, as derived by Knödlseder (2000). Objects 1 and 2, which are at a projected separation of $2.3 \mathrm{pc}$, are both located within the Cyg OB2 core radius. They seem to be part of the very nucleus of the association.

Arguing in favour of their nature as open clusters are $(i)$ the CMDs which are well-defined and different from the field sequences, (ii) the star density profiles indicating important excesses over the background and (iii) the background-subtracted luminosity functions are normal down to A0 stars (completeness uncertainties do not allow inferences on lower masses). Deeper and higher angular resolution photometry is crucial to clarify these issues. However, even assuming that their luminosity functions have a cutoff near A0, the two objects would still be very interesting aggregates of high and intermediatemass stars, in a region where low-mass star formation would have been inhibited. Their angular diameters of $4^{\prime}$ and $5^{\prime}$, at a distance of $1.8 \mathrm{kpc}$, convert to linear diameters of 2.1 and $2.6 \mathrm{pc}$, respectively, comparable to those of infrared embedded clusters (Bica et al. 2003).

\section{Concluding remarks}

We optically detected two new open cluster candidates located in Cygnus while inspecting the structure of the association Cyg OB2 with Digitized Sky Survey images. Fundamental parameters for the objects have been derived using the 2MASS second incremental release catalogue. Both objects are young ( $t \approx 1-4 \mathrm{Myr})$ and appear to be at the same distance to the Sun $\left(d_{\odot}=1.8 \pm 0.2 \mathrm{kpc}\right)$. Object 1 is more absorbed $\left(A_{V}=\right.$ $6.6 \pm 0.2)$ than Object $2\left(A_{V}=5.6 \pm 0.2\right)$. Both objects are closely projected and seem to form a physical pair in the association core, likewise Car OB1 with $\operatorname{Tr} 14 / \operatorname{Tr} 16$. The luminosity functions are normal down to A0 stars, and deeper photometry is necessary to reach lower-mass stars and verify whether they are typical open clusters or interesting aggregates of high and intermediate-mass stars. In case of normal luminosity functions throughout, their masses would be intermediate between those of Trapezium and NGC 3603. As a result of the inspection of $312 K_{\mathrm{S}}$ images of the region, another uncatalogued object was found with CMD morphology suggesting an intermediate-age cluster, and deeper photometry is necessary to verify that possibility.

Acknowledgements. We thank the referee Dr. R. D. Jeffries for important remarks. This publication makes use of data products from the Two Micron All Sky Survey, which is a joint project of the University of Massachusetts and the Infrared Processing and Analysis Center/California Institute of Technology, funded by the National Aeronautics and Space Administration and the National Science Foundation. We employed catalogues from CDS/Simbad (Strasbourg) and Digitized Sky Survey images from the Space Telescope Science Institute (U.S. Government grant NAG W-2166) obtained using the extraction tool from CADC (Canada). We also made use of the WEBDA open cluster database. We acknowledge support from the Brazilian Institutions CNPq and FAPESP. CMD acknowledges FAPESP for a post-doc fellowship (Proc. 00/11864-6).

\section{References}

Alter, G., Ruprecht, J., \& Vanysek, V. 1970, in Catalogue of star clusters and associations + supplements, 2nd ed. (Budapest: Akad. Kiado)

Bica, E., Dutra, C. M., \& Barbuy, B. 2003, A\&A, 397, 177

Binney, J., \& Merrifield, M. 1998, in Galactic Astronomy (Princeton, NJ: Princeton University Press), Princeton series in astrophysics, QB857.B522

Comerón, F., \& Torra, J. 1999, A\&A, 349, 605

Comerón, F., \& Torra, J. 2001, A\&A, 375, 539

Comerón, F., Pasquali, A., Rodighiero, G., et al. 2002, A\&A, 389, 874

Delgado, A. J., \& Alfaro, E. J. 2000, AJ, 119, 1848

Dias, W. S., Alessi, B. S., Moitinho, A., \& Lépine, J. R. D. 2002, A\&A, 389, 871

Dickel, H. R., Wendker, H. J., \& Bieritz, J. H. 1969, A\&A, 1, 270

Dutra, C. M., Santiago, B. X., \& Bica, E. 2002, A\&A, 381, 219

Dutra, C. M., \& Bica, E. 2000, A\&A, 359, L9

Dutra, C. M., \& Bica, E. 2001, A\&A, 376, 434

Girardi, L., Bertelli, G., Bressan, A., et al. 2002, A\&A, 391, 195

Humphreys, R. M. 1978, ApJS, 38, 309

Hutchings, J. B. 1981, PASP, 93, 50

Knödlseder, J. 2000, A\&A, 360, 539

Le Duigou, J.-M., \& Knödlseder, J. 2002, A\&A, 392, 869

Lyngå, G. 1987, in Computer based catalogue of open cluster data, 5th ed. (Strasbourg: CDS)

Massey, P., Johnson, K. E., \& DeGioia-Eastwood, K. 1995, ApJ, 454, 151

Massey, P., \& Thompson, A. B. 1991, AJ, 101, 1408

Melnick, J., Tapia, M., \& Terlevich, R. 1989, A\&A, 213, 89

Mermilliod, J. C. 1996, in The origins, evolution, and destinies of binary stars in clusters, ASP Conf. Ser., 90, 475

Moffat, A. F. J. 1983, A\&A, 124, 273

Odenwald, S. F., \& Schwartz, P. R. 1993, ApJ, 405, 706

Prosser, C. F., Stauffer, J. R., Hartmann, L., et al. 1994, ApJ, 421, 517

Schlegel, D. J., Finkbeiner, D. P., \& Davis, M. 1998, ApJ, 500, 525

Schulte, D. H. 1958, ApJ, 128, 41

Skrutskie, M., Schneider, S. E., Stiening, R., et al. 1997, in The Impact of Large Scale Near-IR Sky Surveys, ed. F. Garzon, N. Epchtein, A. Omont, B. Burton, \& P. Persi (The Netherlands: Kluwer), 210, 187

Soares, J. B., \& Bica, E. 2002, A\&A, 388, 172

Torres-DodGen, A. V., Carroll, M., \& Tapia, M. 1991, MNRAS, 249, 1

Vrba, F. J., Coyne, G. V., \& Tapia, S. 1993, AJ, 105, 1010

Walborn, N. R. 1973, ApJ, 180, L35

Wendker, H. J. 1970, A\&A, 4, 378 